\title{
Metabolic engineering of the tryptophan and phenylalanine biosynthetic pathways in rice
}

\author{
Kyo Wakasa ${ }^{1, *}$, Atsushi Ishihara ${ }^{2}$ \\ ${ }^{1}$ Faculty of Agriculture, Tokyo University of Agriculture, Atsugi, Kanagawa 243-0034, Japan; ${ }^{2}$ Faculty of Agriculture, \\ Tottori University, Tottori 680-8553, Japan \\ *E-mail: k3wakasa@nodai.ac.jpＴel \& Fax: +81-046-270-6504
}

Received October 8, 2009; accepted November 2, 2009 (Edited by Y. Tozawa)

\begin{abstract}
Aromatic amino acids function as building blocks of proteins and as precursors for secondary metabolism. To obtain plants that accumulate tryptophan (Trp) and phenylalanine (Phe), we modified the biosynthetic pathways for these amino acids in rice and dicot species. By introducing a gene encoding a feedback-insensitive anthranilate synthase (AS) alpha subunit, we successfully obtained transgenic plants that over-accumulated Trp. In addition, we found mutant calli that accumulated Phe and Trp at high concentrations. The causal gene (mtrl-D) encoded an arogenate dehydratase (ADT)/prephenate dehydratase (PDT) that catalyzes the final reaction in Phe biosynthesis. The wild-type enzyme was sensitive to feedback inhibition by Phe, but the mutant enzyme encoded by $m$ tr $1-D$ was relatively insensitive. Further, detailed analysis of downstream secondary metabolism from Trp in rice revealed that the Trp pathway, by producing serotonin, is involved in the defense response against pathogenic infection. Based on these findings we propose that the reactions catalyzed by AS and ADT are critical regulatory points in the biosynthesis of Trp and Phe, respectively. In addition, detailed characterization of transgenic lines that accumulate these aromatic amino acids provided new insights into the regulation of downstream secondary metabolism, translocation of aromatic amino acids, and effects of accumulation of aromatic amino acids on various agronomic traits.
\end{abstract}

Key words: Aromatic amino acids, transgenic rice, Oryza sativa, secondary metabolism.

Tryptophan (Trp) and phenylalanine (Phe) are essential aromatic amino acids that animals cannot synthesize and are dependent on plants for their supply. In particular, Trp contributes to the nutritional quality of plant-based foods, and, along with lysine, methionine and threonine, it is used to supplement animal feeds. The other aromatic amino acids, Phe and tyrosine (Tyr), are used for the production of the low-calorie sweetener aspartame and the anti-Parkinson's disease drug L-dopa, respectively. The biosynthetic pathways for aromatic amino acids and their regulation have been extensively explored in bacteria because of their utility in the food and drug industry. In plants, aromatic amino acids are the precursors for a large variety of secondary metabolites including indole alkaloids, phenylpropanoids, flavonoids, and the phenolic polymer, lignin. One of plant growth regulators, indole-3-acetic acid (IAA), has also been shown to be biosynthesized from Trp. Thus, aromatic amino acids also play important roles in plant defense and development.

In bacteria, fungi and plants, the three aromatic amino acids, Trp, Phe and Tyr, are synthesized from a common precursor, chorismate that originates from the shikimate pathway (Figure 1). In bacteria, this pathway is almost exclusively used to produce aromatic amino acids for protein synthesis but, in higher plants, this pathway leads to the production of numerous aromatic secondary metabolites. The importance of this pathway in plants is indicated by the fact that about $20 \%$ of the carbon fixed by photosynthesis flow through this pathway. These facts strongly suggest that the modification of the shikimate and its derivative (or downstream) pathways may lead to dramatic changes in secondary metabolism.

The enzymatic reactions in the biosynthetic pathway for aromatic amino acids seem to be identical in prokaryotes and eukaryotes but they apparently differ in the regulatory mechanisms for these pathways. For example, 3-deoxy-D-arabino-heptulosonate 7-phosphate (DAHP) synthase, the enzyme that catalyzes the first reaction in the shikimate pathway, is not feedback inhibited by any of the aromatic amino acids in plants, but, each of the three DAHP synthase isoenzymes are

Abbreveations: AS, anthranilate synthase; ADT, arogenate dehydratase; Asn, asparagine; Asp, aspartate; PDT, prephenate dehydratase; Phe, phenylalanine; Trp, tryptophan; Tyr, tyrosine.

The editing of this review was supported by the invited editor, Dr. Yuzuru Tozawa (Cell-Free Science and Technology Research Center, Ehime University). The chief editor deeply appreciates his assistance.

This article can be found at http://www.jspcmb.jp/ 


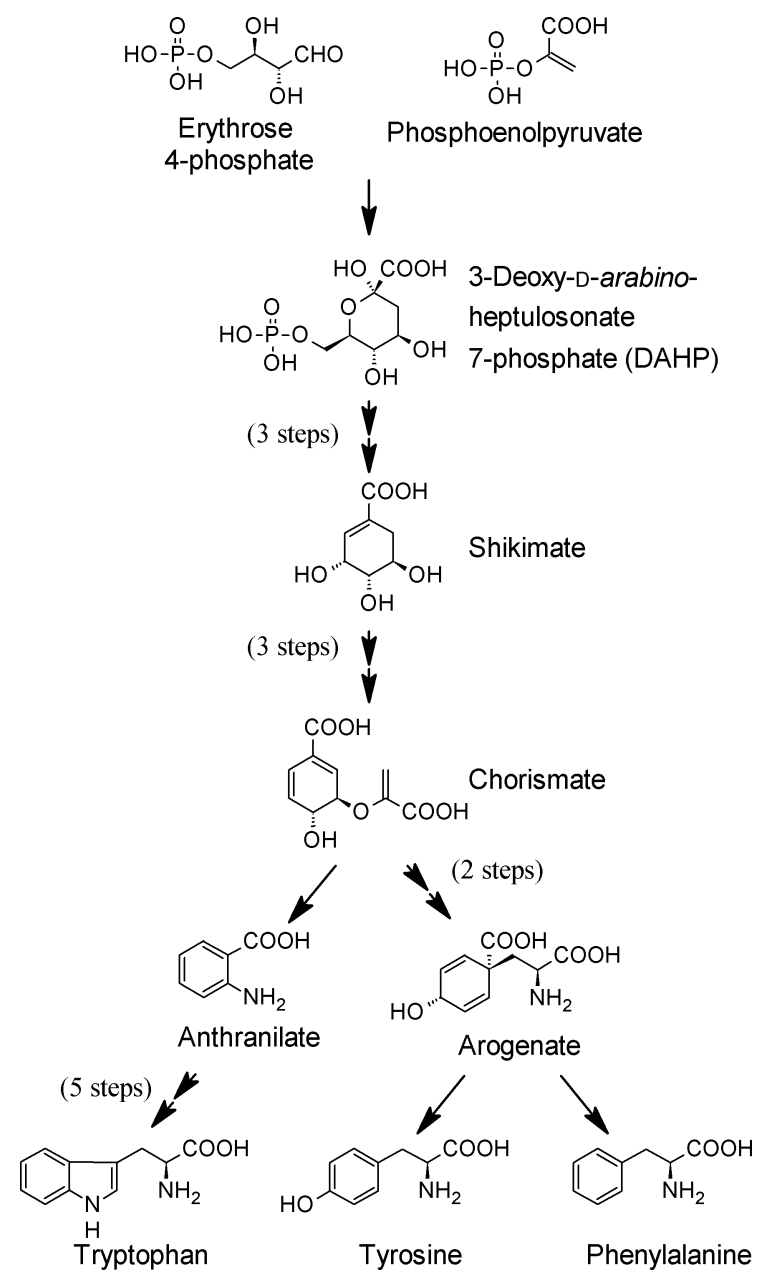

Figure 1. Biosynthetic pathways for aromatic amino acids originate from the shikimate pathway and share chorismate as a common precursor.

sensitive to Phe, Tyr or Trp in Escherichia coli (Hermann 1995). Thus, to improve the production or accumulation of the essential aromatic amino acids, Trp and Phe, in plants, it is necessary to determine and manipulate the unique regulatory points in their biosynthetic pathways.

Chorismate, the last common intermediate of Trp and Phe, is biosynthesized from phosphoenolpyruvate and erythrose 4-phosphate by the shikimate pathway. Anthranilate synthase (AS) converts chorismate into anthranilate in the Trp biosynthetic pathway, whereas in the Phe/Tyr pathway, chorismate mutase (CM) acts on chorismate to produce prephenate. In bacteria, these enzymes are regulated by repression and attenuation during transcription and translation, respectively, and by feedback inhibition of enzyme activity. Plants show a similarly complex regulation. Feedback regulation of both enzyme activities has been reported in various plants as well (Figure 2) (Poulsen et al. 1993; Li and Last 1996; Goers and Jensen 1984; Mobley et al. 1999). In addition, the expression of the genes encoding AS and $\mathrm{CM}$ is also regulated at the transcription stage.

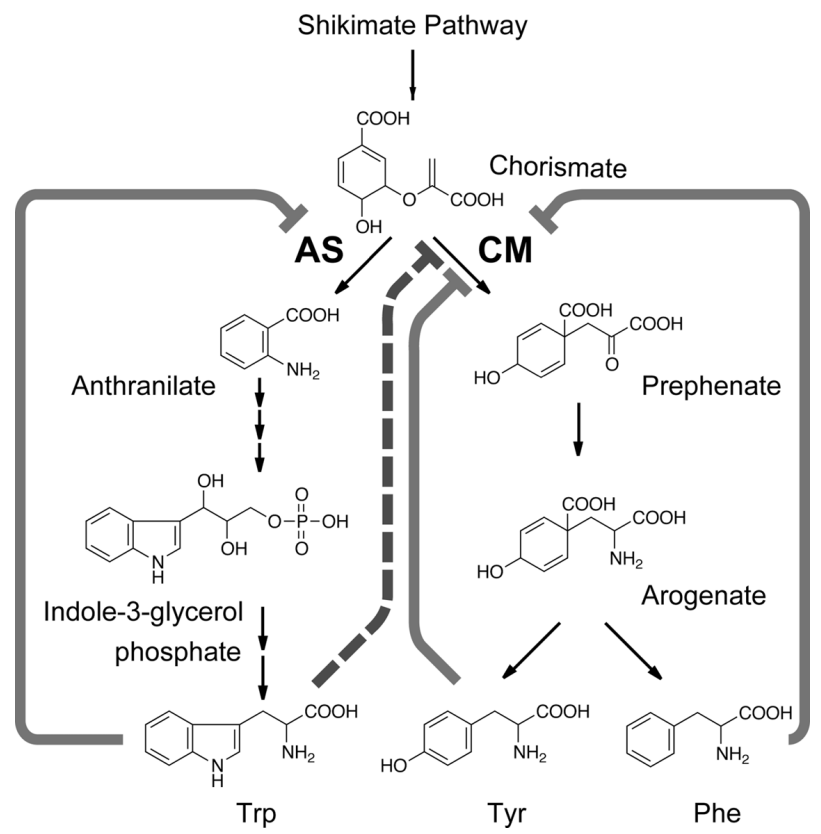

Figure 2. Feedback regulation of anthranilate synthase and chorismate mutase by aromatic amino acids. Gray lines indicate feedback inhibition, and a dashed line indicates feedback activation.

Exogenous stimuli such as pathogenic infection, elicitation and wounding affect their expression levels (Niyogi and Fink 1992; Bohlman et al. 1995, 1996; Kuroki and Conn 1989; Mobley et al. 1999). Differential expression of isozymes is another factor controlling these biosynthetic pathways. All of the species examined so far have been shown to possess at least two isozymes for AS and CM (Niyogi and Fink 1992; Bohlman et al. 1995; Mobley et al. 1999). These multiple layers of regulatory mechanisms suggest that the regulation of aromatic amino acids and optimization of aromatic amino acid levels are important for plants.

Prephenate is further converted to Phe via arogenate by two different enzyme reactions catalyzed by prephenate aminotransferase and arogenate dehydratase (ADT). ADT activity has been detected in several plant species and shown to be inhibited by Phe, suggesting that this enzyme is an additional regulatory step in Phe synthesis (Jung et al. 1986; Siehl and Conn 1988). Based on the detection of prephenate dehydratase (PDT) activity in Arabidopsis, Warpeha et al. (2006) proposed a different route that involved the conversion of prephenate via phenylpyruvate to Phe. Thus, the biosynthetic route from prephenate and its regulatory role in Phe synthesis is still unclear.

This article describes the results of our efforts to modify the biosynthesis of Trp and Phe through the manipulation of key regulatory mechanisms. The acquisition of new transgenic and mutants lines that accumulate aromatic amino acids at high concentrations provided an opportunity to analyze the effects of 
overproduction of aromatic amino acids on agronomic traits, secondary metabolism, and defense reaction against pathogenic infection in plants.

\section{Manipulation of Trp content}

Mutations that result in auxotrophy for aromatic amino acids or resistance to their lethal analogs have been used for increasing the content of lysine, Trp and Phe in bacteria. Since it is difficult to establish auxotroph mutant in plants, mutants resistant to amino acid analogs have been widely used for increasing amino acid levels. The accumulation of Trp was first demonstrated in tobacco mutant cells resistant to 5-methyltryptophan (5MT), a Trp analog (Widholm 1972). In this mutant, AS activity was insensitive to high Trp levels. The causal gene was identified in an Arabidopsis mutant resistant to Trp analog 6-methyltryptophan. In this mutant, point mutation of a single amino acid in the AS $\alpha$ subunit led to insensitivity to negative feedback regulation and a consequent 3-fold increase in Trp content ( $\mathrm{Li}$ and Last 1996).

AS consists of $\alpha$ and $\beta$ subunits in plants and may function as $\alpha_{2} \beta_{2}$ (Coruzzi and Last 2000). The $\alpha$ subunit is deemed to play a crucial role for feedback inhibition; it has a Trp binding site that enables allosteric regulation. Complementary DNAs encoded by AS $\alpha$ subunit genes have been isolated from Arabidopsis (ASA1 and ASA2; Niyogi and Fink 1992), Ruta graveolens (AS $\alpha 1$ and $A S \alpha 2$; Bohlmann et al. 1995), tobacco (ASA2; Song et al. 1998) and rice (OASA1 and OASA2; Tozawa et al. 2001). A phylogenetic tree based on the deduced amino acid sequences revealed a close evolutionary relationship among Arabidopsis ASA2, R. graveolens AS $\alpha 2$, tobacco ASA 2 and rice OASA1; however, Arabidopsis ASA1, $R$. graveolens AS $\alpha 1$ and rice OASA2 were quite diverse (Tozawa et al. 2001). The two groups of AS $\alpha$ subunits also differ in their sensitivity to Trp and transcription regulation. Enzymes encoded by $A S \alpha 1$ and tobacco $A S A 2$ are insensitive to high levels of Trp. The synthesis of ASA1 mRNA in Arabidopsis is induced by wounding and bacterial infection whereas elicitor treatment induced the transcription of $O A S A 2$ in rice. These findings suggest a role of these AS $\alpha$ subunits in alkaloid biosynthesis and/or in the production of plant defense substances. In fact, transcription of rice $O A S A 2$ was induced after inoculation with Bipolaris oryzae (Ishihara et al. 2008). Expression of the other AS $\alpha$ subunit genes in Arabidopsis ( $A S A 2)$ and rice $(O A S A 1)$ is not induced by exogenous stimuli and is at a lower level than those of $A S A 1$ and $O A S A 2$ genes, respectively, suggesting that they are involved in the maintenance of basal Trp levels.

As mentioned above, Arabidopsis ASA1 is apparently involved in the production of secondary metabolites, while rice OASA1 seems to be a key enzyme in Trp biosynthesis. However, the substitution of aspartate (Asp) to asparagine (Asn) at corresponding residues both in ASA1 (at 341) and OASA1 (at 323) commonly resulted in the insensitivity of AS to negative feedback regulation by Trp, and consequently in Trp accumulation and 5MT resistance (Li and Last 1996; Tozawa et al. 2001). This Asp residue at 323 of OASA1 and 341 of ASA1 is well conserved in other plant AS $\alpha$ subunits.

The mutant OASA1 protein that has a substitution of Asp by Asn at 323 was labeled as OASA1D. The gene $(O A S A 1 D)$ encoding this mutant enzyme was introduced to rice, and two homozygous lines (HW1 and HW5) were established (Wakasa et al. 2006). Leaves of 8-d-old seedlings from these lines accumulated Trp up to 46- and 30 -fold greater than those in the control (Dubouzet et al. 2007). Seeds of field grown plants accumulated Trp up to 311- and 193-fold more than those in control seeds (Wakasa et al. 2006). This mutated gene was introduced into several dicotyledonous species as well. Elevated Trp content was observed in potato tuber (Yamada et al. 2004), soybean seeds (Ishimoto et al. 2009) and Arabidopsis plants (Ishihara et al. 2006). These findings unequivocally indicate that nullification of the feedback regulation of AS is critical for the accumulation of Trp in plants.

The analysis of Trp in OASA1D transgenic lines also provided a new insight into Trp translocation. In transgenic rice overexpressing $O A S A 1 D$, the distribution of Trp was uneven in the whole plant in spite of the uniform expression of the OASAID gene under the control of ubiquitin promoter. The highest Trp level was always found in the youngest leaf of OASA1D transgenic rice. In 1-week-old HW5 seedlings, the Trp content in the first (youngest) leaf was very high $(5,300 \mathrm{nmol} \mathrm{g}$ fresh weight ${ }^{-1}$ ). When the second and third leaf blades were expanded in 2-week-old seedlings, the highest amount of Trp (about 2,700 nmol g fresh weight ${ }^{-1}$ ) was then found in the third (youngest) leaf. The levels of Trp in the first leaf and second leaf decreased to the level of Nipponbare at this stage. Four weeks after germination, the highest level of Trp (about 1,800 nmol $\mathrm{g}$ fresh weight ${ }^{-1}$ ) was again found in the youngest leaf (fourth leaf). A similar trend was observed in Arabidopsis seedlings expressing $O A S A 1 D$ gene (Ishihara et al. 2006). No differences in AS activity and sensitivity to Trp feedback inhibition were observed among these tissues. However, a feeding experiment with radiolabeled Trp clearly demonstrated one-way Trp movement from old to young leaves in both wild-type and $O A S A 1 D$ transgenic lines (Matsuda et al. in press). This uneven distribution of Trp may be a manifestation of the large capacity for Trp translocation in wild-type rice plants. Trp translocation may be required to salvage Trp from leaves undergoing senescence due to age or induced by mechanical damage or pathogenic infection. 
In OASA2, Asp at the position corresponding to the 323 residue in OASA1 is not conserved, but substituted by Asn. However, OASA2 is feedback sensitive, and its overexpression did not result in the alteration of sensitivity of enzyme AS and Trp content (Tozawa et al. 2001). Kanno et al. (2005) used a structure-based in vitro engineering method to investigate the role of specific amino acid residues involved in feedback inhibition in OASA2. They used in vitro mutagenesis to generate 36 mutant OASA2 sequences and evaluated the corresponding translation products in a wheat-embryo cell-free system. Examination of enzyme functions of these proteins revealed six mutants with elevated catalytic activity and five mutants with enhanced tolerance to feedback inhibition by Trp. Four of these five mutants, however, showed reduced enzyme catalytic activities. The simultaneous introduction of two types of mutations resulted in a remarkable improvement of catalytic activity without a loss of feedback insensitivity. Rice cells expressing these mutant OASA2 genes exhibited improvement in free Trp content (Kanno et al. 2005). A cell line expressing one of these mutant OASA2 genes accumulated $400 \mathrm{X}$ more Trp compared to Nipponbare callus (unpublished data).

\section{Influence of overproduction of Trp to metabolic profiles}

Because Trp and intermediates in the Trp biosynthetic pathway are precursors for a diverse range of secondary metabolites, the influence of overproduction of Trp on the metabolic profile and plant phenotype are of great interest. In addition, alteration of Trp biosynthesis could affect the metabolism of aromatic compounds in various ways (Ishihara et al. 2007). For example, feedback activation of CM (Mobley et al. 1999) by accumulated Trp may result in the enhanced production of Phe and Tyr. Alternatively, the increased conversion of chorismate by AS may decrease the metabolic flux into the CM branch of the shikimate pathway. Thus, nontargeted analysis of aromatic compounds was performed by reversed-phase HPLC coupled with photodiode array detection over a wavelength range of 190-400 nm. However, no apparent marked accumulation of components other than Trp was detected in the seeds and leaves of $O A S A 1 D$ transgenic rice (Wakasa et al. 2006; Dubouzet et al. 2007; Matsuda et al. in press). The increase of Trp (164.4-fold) in seeds of HW lines was accompanied by increases in the amount of other free amino acids and in the total amount of free amino acids (4.2-fold in HW1 and 2.9-fold in HW5). This suggests the presence of a regulatory mechanism for overall amino acid synthesis in response to Trp accumulation (Wakasa et al. 2006).

A non-target metabolic profiling using LC-MS was also applied to seedlings of HW1 and HW5 (Matsuda et al. in press). The seedlings accumulated Trp at a high concentration without marked effects on the amounts of other major metabolites. However, we detected an increase in 26 peaks in addition to Trp. The chemical components of these 26 peaks were structurally characterized by LC-MS/MS, and eight of them were assigned to five metabolites, indole-3-propionic acid, 1,2,3,4-tetrahydro- $\beta$-carbolin-3-carboxylic acid, $\gamma$ glutamyltryptophan, and two glucosides of indole compounds. Compared to Trp, the intensities of the 26 peaks were much weaker. Thus, Trp is not actively metabolized into these compounds in transgenic rice.

In addition to the non-target metabolic profiling, targeted analysis of indole amines (tryptamine and serotonin) was performed. The analysis detected modest changes in tryptamine and serotonin contents in rice calli, seeds and plants. For instance, LC-MS/MS analysis revealed that 8-day-old $O A S A 1 D$ seedlings had higher levels of tryptamine and serotonin than Nipponbare seedlins (Dubouzet et al. 2007). The amounts of tryptamine and serotonin in the transgenic lines were 2- to 4-fold larger than those in the wild type, but their respective amounts were only $0.06 \%$ and $0.7 \%$ of Trp. Thus, targeted analysis also revealed that Trp overproduction had a limited effect on downstream metabolism. Serotonin accumulation will be discussed in the relationship with defense mechanisms in a later section.

\section{Influence of overproduction of Trp to phenotype in rice}

Targeted and non-targeted analyses of metabolites have clearly shown that transgenic rice overexpressing $O A S A 1 D$ gene has no dramatic change in aromatic amino acid metabolites except Trp. However, the free IAA contents in the seeds of HW1 and HW5 were approximately double that of Nipponbare (Wakasa et al. 2006). Higher IAA may affect plant phenotype because auxin plays an important role in the regulation of plant growth and development.

Two homozygous lines of OASAID transgenic rice, HW1 and HW5, were characterized for stability of Trp accumulation and agronomic traits under field condition (Wakasa et al. 2006). In a two-year trial, HW1 and HW5 grown in the field stably accumulated free Trp in their seeds (Wakasa et al. 2006: Terakawa et al. submitted). The free Trp content of seeds of field-grown HW lines was double that of seeds of plants grown in the greenhouse. This increase of free Trp was not accompanied by an increase of the total Trp; the latter was stable under different growth conditions and the seeds from HW plants had almost double the amount of total Trp compared to Nipponbare. 
Days to heading and morphological traits such as culm length, panicle length and the number of tillers of HW lines were similar to the wild-type Nipponbare plants. Values for culm length fluctuated when the lines were grown in an isolated paddy field (2003) or normal paddy field (2004). Although most agronomic traits other than the culm length appeared similar to Nipponbare, differences in traits related to seed productivity were apparent between the transgenic lines, especially HW1, and the wild-type Nipponbare. Spikelet fertility and average spikelet number per panicle in both transgenic lines were significantly lower than those of Nipponbare. These reductions likely contributed to a decrease in harvested seed weight. In the case of seeds from greenhouse-grown plants, the germination rates of HW lines were similar to that of Nipponbare. However, it was greatly reduced in seeds from field grown HW1 plants compared to Nipponbare, indicating that the growth condition may have markedly influenced seed germination rate. This may be causally related to accumulation of Trp and/or IAA: Under field conditions, a higher content of free Trp was observed in seeds of both HW lines compared to those grown in the greenhouse, and seeds of HW1 always had higher contents of free and total Trp than HW5.

The high level of free Trp in HW1 might be a reason for the decrease of spikelet fertility and impaired germination. Higher levels of IAA were detected in the seeds of HW lines and this may have a bearing on the reduction of spikelet fertility and germination percentage in HW lines. However, the amount of total IAA was slightly higher in the seeds of HW5 than in those of HW1. Both Trp and IAA might have an additive effect on seed productivity, but it was difficult to distinguish the effect of each factor. Inhibition of germination by IAA and Trp was also observed in wheat embryos (Ramaih et al. 2003).

The spikelet number per panicle is another important factor related to the productivity in cereals and it is determined at an early stage of development of the inflorescence apex. In contrast to OASA2, whose mRNA transcripts were observed in all organs of leaves, roots and immature panicles, OASAl was expressed in immature panicles, but not in leaves and roots (Tozawa et al. 2001). This organ-specific expression of OASA1 suggests a specific function of the gene product at this stage of growth. Accordingly, its overexpression may affect the development of the inflorescence.

Spikelet fertility in both HW lines improved when grown in a normal paddy field (Terakawa et al. submitted). Almost the same spikelet fertility was observed in the HW5 line and Nipponbare. This suggests that culture condition(s) such as the amount of fertilizer applied and water balance may improve fertility in rice plants over-accumulating Trp.

\section{Phe biosynthesis is also controlled by feedback regulation}

The regulation of Trp biosynthesis has been relatively well characterized as a result of the discovery of several mutants that were resistant to Trp analogs and accumulated intermediates in the Trp biosynthetic pathway. Our results confirmed that AS is a critical regulatory point in Trp biosynthesis and the feedbackinsensitive form of the AS enzyme is crucial to the accumulation of Trp in rice, soybean, potato and Arabidopsis. In contrast to Trp, there are no other mutant plants that accumulate Phe aside from the rice Mtr1 mutant that accumulates Phe and Trp (Wakasa and Widholm 1987).

The Mtr1 line was originally generated from callus tissue resistant to 5MT; it had a single dominant mutation and showed high Trp levels. We originally suspected that the responsible mutation was in the AS genes. However, feedback inhibition of AS activity by Trp did not differ between Mtr1 and the wild-type variety Norin 8 (Yamada et al. 2008). The metabolic profile of Mtr1 calli revealed accumulation of multiple compounds at high levels relative to Norin 8 (Yamada et al. 2008). Chemical structures of those eight compounds were determined by spectroscopic analyses. Among them, seven were phenylpropanoids and one was an indole alkaloid that was identical to an indole derivative detected in transgenic rice calli that over-accumulated Trp (Morino et al. 2005). Since phenylpropanoids originate from Phe, these findings suggested that the altered regulation of Phe biosynthesis caused the observed phenotypes in the mutant.

The candidate for Mtr1 mutant gene ( $m$ tr $1-D)$ that had been annotaited as a putative PDT was isolated by positional cloning (Yamada et al. 2008). The mtrl-D gene contained a single base substitution at nucleotide position 893 as compared to the corresponding genes in Norin 8 and Nipponbare. The overexpression of the mutant gene in rice calli conferred manifold characteristics to the Mtr1 callus. Calli overexpressing mtrl-D showed elevated levels of Phe, Trp, phenylpropanoids and an indole alkaloid, and was resistant to 5MT treatment. Biochemical analysis of the proteins encoded by the wild type PDT or $m$ tr $1-D$ gene revealed that the protein catalyzed both PDT and ADT reactions. PDT catalyzes the conversion of prephenate to Phe, while ADT catalyzes the conversion of arogenate to Phe. These reactions are part of two different routes in Phe synthesis from prephenate via arogenate or phenylpyruvate. In the former, Phe is synthesized from prephenate via arogenate, while the intermediate in the other route is phenylpyruvate. In plants, the former route has been suggested to be the main route (Siehl and Conn 1988; Jung et al. 1986), while in bacteria, the latter route 
is functional (Nester and Jensen 1996; Im and Pittard 1971; Hall et al. 1982). The enzyme encoded by Mtrl gene preferred arogenate as substrate and its catalytic efficiency with arogenate is about 10 -fold higher than that with prephenate, suggesting that the route that produces arogenate is preferred in rice as well. We call the enzyme encoded by the Mtrl gene as ADT/PDT based on its enzyme activity. The enzyme activity of both wild-type ADT and PDT was inhibited by Phe, whereas the mutant enzyme encoded by $m$ tr $1-D$ showed a reduced sensitivity to Phe. It has been suggested that the regulation of $\mathrm{CM}$ is important to control the flux from chorismate to aromatic amino acids in the shikimate pathway (Figure 2). However, our observations indicate that rice ADT/PDT is critical for regulating the size of Phe pool in plant cells.

\section{Effects of Phe accumulation to plant phenotype}

Both calli and seedlings were resistant to 5MT, but we investigated the inheritance of the mutation using resistance of seedlings to $1 \mathrm{mM} 5 \mathrm{MT}$. When the sensitivity of 204 R4 progeny families (produced by selfing the resistant R3 plant families) was tested, all of the R3 plants families showed segregation for resistance and sensitivity to 5MT. The progenies of crossed hybrids between resistant Mtr1 and wild-type plant (Norin 8) also showed a similar segregation pattern. The possible reasons why no homozygous resistant plant was observed were explored: No chromosomal aberration was observed in the resistant Mtr1 plant (Wakasa and Widholm 1987, 1991). Resistant calli derived from rice pollen of resistant Mtr1 plants (heterozygous for $m$ tr 1-D) were obtained by anther culture and the proportion of resistant pollen deduced from the number of resistant calli was near normal. Moreover, acetocarmine-staining of pollen revealed no differences in fertility in 5MTresistant and sensitive plants (Wakasa and Widholm 1991). If the homozygous zygotes for $m t r l-D$ gene were lethal, spikelet fertility or germination frequency should be below $75 \%$ in resistant Mtr1 plants (heterozygous). However, because spikelet fertility was always above $75 \%$ and the germination frequency was higher than $80 \%$, it was unlikely that apparent lethality occurred in homozygous zygotes or seeds.

The agronomic traits of plants resistant or sensitive to 5MT were compared (Fukuchi et al. 1990). Resistant plants accumulating Trp and Phe presented some characteristics similar to HW lines. They showed slightly shorter culms and panicles in comparison to sensitive plants as HW lines showed. The number of spikelet per panicle was also different in resistant and sensitive plants. The spikelet number was 40 to 50 grains less in resistant plants than in sensitive plants. The spikelet fertility was slightly smaller in resistant plants (80 to $90 \%$ ) than in sensitive ones (91 to $95 \%$ ). Sensitive plants produced from resistant parents were almost identical to the control variety Norin 8 . Germination rates of resistant lines in a medium with 5MT were normal (87 to $97 \%$ ) but slightly lower than in sensitive lines (95 to 100\%) (Wakasa and Widholm 1987).

The Mtr1 mutants displayed the same short culm and reduced spikelet number that were observed in transgenic rice accumulating Trp. The main difference between the HW lines and the Mtr1 mutants was in trait inheritance: only the heterozygous zygote for $m$ trl-D could grow and produce seed. Compared to HW lines, the Mtr1 mutants showed slightly better germination frequency and spikelet fertility. It is interesting that the Mtr1 callus contained a high level of secondary metabolites including phenylpropanoids and indole alkaloid, but these did not have a significant effect on the rest of the plant's morphological and other agronomic characteristics. This suggests that, compared to callus tissue, the whole plant may have a different homeostatic response to marked changes in its metabolomic profile.

The mutation in Mtr1 was shown to reside in an enzyme associated with Phe biosynthesis. It is likely that the increase in Phe and phenylpropanoid levels is the consequence of insensitivity of the mutant ADT/PDT to feedback inhibition by Phe. However, it is difficult to explain how the accumulation of Phe results in both Trp accumulation and 5MT resistance. The feeding experiments with $300 \mu \mathrm{M}$ Phe in wild-type calli and $600 \mu \mathrm{M}$ Phe to seedlings demonstrated that excess Phe conferred 5MT resistance. In addition, feeding of $2 \mathrm{mM}$ Phe resulted in Trp accumulation in rice seedlings. These results clearly indicated the relation between Phe accumulation and Trp pool size.

\section{Defensive role of Trp pathway-derived secondary metabolism}

Aromatic amino acids are converted to secondary metabolites and many of these have been implicated in the chemical defense mechanisms in plants (Dixon and Paiva 1995). Phe-derived secondary metabolites include phenylpropanoids, coumarin, flavonoids, and isoflavonoids, etc. Their accumulation is either constitutive or inducible depending on the compound and plant species. The early biosynthetic steps for these metabolites are shared by biosynthetic pathways for phenolic polymers like lignin and suberin that may play a significant role in the defensive response to pathogenic infection in plants (Nicholson and Hammerschmidt 1992).

The ecological and physiological functions of Trpderived secondary metabolites have not attracted as much attention as the Phe-derived metabolites although 
some Trp-derived alkaloids have been investigated as anticancer agents. In the grass family, however, multiple Trp-pathway derived secondary metabolites have been found in relation to the defense against pathogens. For example, avenanthramides in oats (Mayama et al. 1981; Ishihara et al. 1999), benzoxazinones in wheat, rye and maize (Niemeyer 1988; Frey et al. 1997; Nomura et al. 2003) and gramine in barley (O'Donovan and Leete 1963; Sepulveda and Corcuera 1990) have been characterized as the factors controlling resistance of the host species against pathogenic infection. In spite of the presence of Trp-pathway derived metabolites in related species, no such compounds have been identified in rice. Thus, we started investigating these compounds in rice.

We inoculated rice leaves with conidia of rice brown spot fungus, Bipolaris oryzae, and analyzed the subsequent changes in Trp-derived metabolites by HPLC and LC-MS/MS. Serotonin (5-hydroxytryptamine) content dramatically increased in response to pathogenic infection in rice leaves (Ishihara et al. 2008). The radiolabeled serotonin was incorporated into the cell wall fraction in the lesion, and peroxidase activity that accepts serotonin as a substrate was upregulated after pathogenic infection. Thus, serotonin (and/or its derivatives) may be deposited in the cell walls and subjected to oxidative polymerization and cross-linking catalyzed by inducible peroxidases to form a phenolic barrier against fungal invasion. A mutant line $(s l)$ of rice was reported to accumulate tryptamine at a high concentration in the lesion formed after infection with Magnaporthe grisea (Ueno et al. 2003). We analyzed the contents of tryptamine and serotonin in the mutant leaves inoculated with $B$. oryzae, and found that this mutant accumulated tryptamine but not serotonin. In addition, the lesion in the mutant leaves lacked deposition of brown materials, suggesting that the materials observed in infected leaves of wild-type plants were formed by serotonin polymerization. The pathway from Trp to polymerization products of serotonin is shown in Figure 3. The elongation of hyphae of $B$. oryzae in the mutant tissues was stimulated in comparison with that in the wild-type leaves. Thus, serotonin accumulation and deposition of brown materials in the cell walls were suggested to be a part of an effective physical defense response against pathogenic infection in rice.

Trp pathway-derived secondary metabolites in the grass family include compounds involved in both inducible (e.g., avenanthramides in oats) and constitutive (e.g., benzoxazinones in wheat, rye and maize, and gramine in barley) chemical defense mechanisms. The reinforcement of the cell walls by serotonin, and their participation in physical defense, represent a novel function of secondary metabolites derived from the Trp pathway. Thus, the scope of the functions of the products of the Trp pathway is almost comparable to that of the

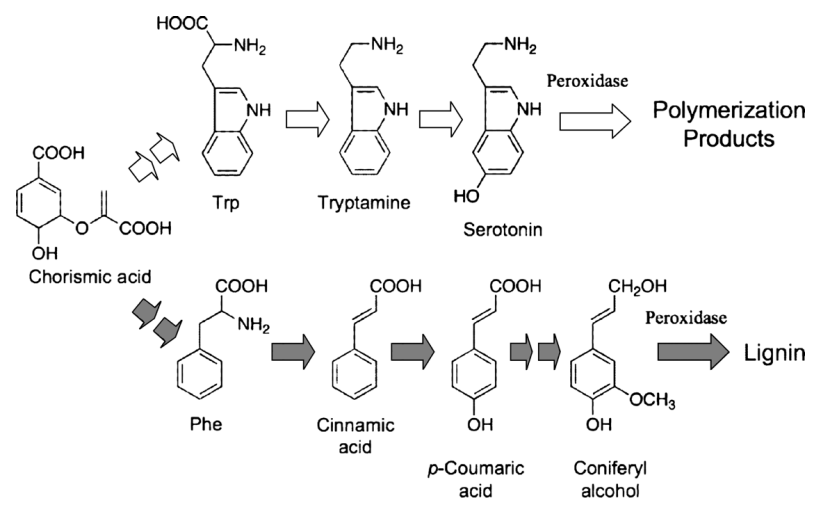

Figure 3. Schematic representation of biosynthesis of polymerization products of serotonin. For comparison, the biosynthetic route for lignin is also presented.

Phe derivatives. Serotonin is a simple indole amine that is biosynthesized from Trp by two steps of enzymatic reactions, and it is widely distributed in the plant kingdom (Ly et al. 2008). The accumulation of serotonin in defense responses may be present in a wide range of plant species. Accordingly, verification of the generality of this response in the monocotyledonous and dicotyledonous species will be interesting from the viewpoint of the evolution of physical defense mechanisms in plants.

The finding of inducible serotonin accumulation in rice leaves provided a unique opportunity to examine the defense response of Trp over-accumulating rice lines, HW1 and HW5. We inoculated conidia of B. oryzae to the leaves of these lines, and analyzed serotonin contents. As shown in Figure 4, however, the amounts of serotonin in these lines were not significantly different from that in the wild type plant. HW1 and HW5 accumulated tryptamine in amounts several times larger than that in the wild type, but the increase rates were quite small considering the Trp amounts in the lines. Serotonin is biosynthesized from Trp via tryptamine, and the first committed reaction is catalyzed by tryptophan decarboxylase (TDC). The small increase in the tryptamine contents in HW1 and HW5 suggests that TDC reaction is under a strict control. In wild type rice seedlings, we found that both TDC transcript level and TDC activity dramatically increased after infection of pathogenic fungi, indicating that TDC reaction is the rate-limiting step in serotonin accumulation (Ishihara et al. 2008). In accordance with this, the simultaneous overexpression of a gene encoding a functional TDC and $O A S A 1 D$ resulted in the accumulation of serotonin at a high concentration (Dubouzet et al. unpublished results). Kang et al. (2007) similarly reported the accumulation of serotonin in the rice seedlings that express a TDC gene under the control of maize ubiquitin promoter.

The effects of expression of the OASAID gene on defensive secondary metabolism were also analyzed in 

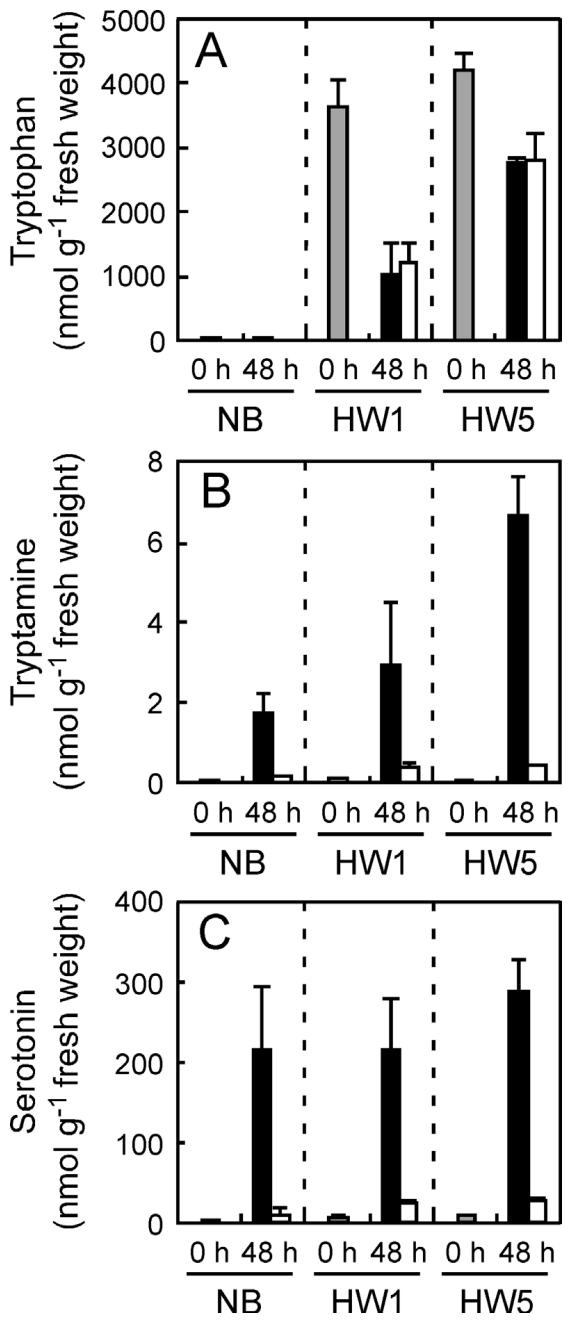

Figure 4. The effects of Trp over-accumulation on the induction of accumulation of tryptamine and serotonin. The third leaves of 12-d-old seedlings of Nipponbare (NB) and transgeninc lines expressing OASA1D (HW1 and HW5) were inoculated with the conidia of $B$. oryzae (black bars) or treated with distilled water (white bars). The amounts at time 0 were expressed by gray bars. After a 48 -h incubation, the leaves were extracted with $80 \%$ methanol. The amounts of Trp (A), tryptamine (B) and serotonin (C) were determined by LCMS/MS analysis in MRM mode. The error bars indicate the standard deviations obtained from at least three replicates.

A. thaliana (Ishihara et al. 2006). This species accumulates indole glucosinolates constitutively, and produces the indole alkaloid phytoalexin camalexin in response to pathogenic attack (Tsuji et al. 1992). These compounds are biosynthesized from Trp, sharing the first committed reaction that is the formation of indole-3acetaldoxime (Hull et al. 2000; Mikkelsen et al. 2000; Glawischnig et al. 2004). We established A. thaliana lines that accumulate various levels of Trp by introducing OASAID, and some lines (high-Trp accumulating lines) accumulated approximately 200 -fold greater amounts of Trp than the wild type. However, these lines showed only slight increases (1.5-fold in shoot and 2.0-fold in root) in the contents of indole glucosinolates. The effect of Trp accumulation on camalexin synthesis was analyzed in leaves infected by Alternaria brassiccola. The amounts of camalexin in the high-Trp accumulating lines were not significantly different from those in the wild type. Thus, the utilization of Trp for the production of secondary metabolites is under strict regulation in A. thaliana as well.

\section{Conclusion}

In the past two decades, we have developed novel technologies to increase aromatic amino acid contents in plants. As described here, we have generated new transgenic and mutant lines that accumulate Trp and Phe at high concentrations through metabolic engineering. In addition, the physiological and biochemical analyses of these lines provided new insights into the regulation of primary and secondary metabolism in plants.

Most importantly, our investigations of the Trp and Phe biosyntheses have led to the identification of key regulatory biochemical steps that are critically important for the control of biosynthetic flux. The introduction of OASA1D gene into monocotyledonous and dicotyledonous species resulted in the enhanced accumulation of Trp. An Arabidopsis mutant (trp5) that expressed AS with relaxed feedback sensitivity had approximately 3-fold higher Trp levels compared to wild-type. We have produced transgenic lines in monocot and dicot species with Trp levels that were several hundred times more than in control plants. This difference is probably attributable to the difference in the feedback sensitivity of the mutated enzyme, considering that the total AS activity in the transgenic lines was not much increased in comparison with that in the wild type. The crude AS enzyme preparation from the trp 5 mutant in Arabidopsis showed a $K_{\mathrm{i}}$ value of $8 \mu \mathrm{M}$ for $\operatorname{Trp}(\mathrm{Li}$ and Last 1996). On the other hand, the $K_{\mathrm{i}}$ value of OASA1D-OASB complex for Trp was reported to be approximately $135 \mu \mathrm{M}$ (Kanno et al. 2004). These findings prove that feedback sensitivity is crucial for the control of Trp content in plants. Matsuda et al. (2007) directly detected the enhanced metabolic flux in OASA1D transgeninc rice cells by dynamic labeling technique.

We have also proven that the relaxed feedback sensitivity of ADT/PDT leads to Phe accumulation. Although ADT activity in crude enzyme has been shown to be regulated by Phe through feedback inhibition (Siehl and Conn 1988), the biochemical significance of this feedback inhibition has not been elucidated. Our experiments unequivocally proved that $\mathrm{ADT}$ is a critical regulatory point in the Phe biosynthesis. The final enzyme in the biosynthetic pathway for Tyr, arogenate dehydrogenase $(\mathrm{ADH})$ has been shown to be sensitive to 
feedback inhibition from Tyr (Rippert and Matringe 2002). It has also been demonstrated that, at least, one of $\mathrm{CM}$ isoforms is sensitive to feedback regulation form Phe and Tyr (Mobley et al. 1999). These findings raise the question about the significance of feedback inhibition of CM in the control of metabolic flow for Phe and Tyr. To answer this question, the development of CM enzyme with relaxed feedback sensitivity and functional evaluation of this gene in transgenic plants are required.

It is of particular interest that the Mtr1 calli accumulated Trp at a high concentration as well as Phe because this finding suggests the presence of interaction between the Phe and Trp branches of the shikimate pathway. The competition for chorismate and the feedback activation of CM by Trp define the interaction between these two derivative branches of the aromatic acid biosynthetic pathway. However, the increase of Trp in Mtr1 calli cannot be explained by these mechanisms. Considering the critical role of feedback inhibition of AS by Trp in the control of Trp pool size, this process may be negatively affected anyhow by increased production of Phe. The elucidation of this mechanism is an interesting and challenging issue to be resolved in the future.

The analysis of secondary metabolism in the $O A S A 1 D$ transgenic plants suggests that Trp metabolism is strictly regulated in plants. This is in sharp contrast to Phe metabolism. Metabolic profiling of the rice Mtr1 callus revealed the accumulation of multiple Phe-derived secondary metabolites at a substantially high concentration (Yamada et al. 2008). The strict control of Trp metabolism might be associated with the fact that Trp is one of the most biosynthetically expensive amino acids, and plants may have developed a mechanism to prevent precious metabolites from unnecessary utilization as suggested by Matsuda et al. (in press). This may also help explain the strict control of Trp biosynthesis by feedback inhibition of AS and the large capacity of translocation of Trp from old to young leaves. Alternatively, the strict control of Trp metabolism might be related to the biosynthesis of IAA. Three different pathways from Trp have been proposed for IAA biosynthesis, and the first intermediates in these pathways are tryptamine, indole-3-acetaldoxime, and indole-3-pyruvate. Thus, the initial compounds for secondary metabolism from Trp are also the intermediates for the IAA biosynthesis. Excess accumulation of these intermediates potentially affects the IAA level, which is an extremely important factor for plant growth regulation. Indeed, the blockage of downstream reaction in indole glucosinolate metabolism by mutation in CYP83B1 results in the overaccumulation of IAA (Bak et al. 2001). To avoid accumulation of excess amount of intermediates that the downstream metabolism cannot deal with, the reaction leading to the formation of these intermediates might be under the strict control.

From the viewpoints of practical utilization of the transgenic lines, it is preferable to eliminate the selection marker gene for the establishment of crop with high content of Trp by genetic engineering. The OASA1D gene confers clear 5MT resistant to plant cells (Tozawa et al. 2001; Yamada et al. 2004). Therefore, transgenic plants can be generated without any other selection marker gene. In fact, the rice plants with only $O A S A 1 D$ gene cassette have been established and they showed elevated Trp levels (Komatsu et al. 2006).

The transgenic rice lines expressing $O A S A 1 D$ are very close to commercial utilization because we have not observed serious agronomic problems in field trials. The strict regulation of Trp metabolism is thought to be suitable for this purpose. In the case of Mtr1 plants, the homozygote of the mutation has not been obtained. Although the reason for this is not clear, fine tuning of the feedback sensitivity of ADT may be effective to establish a line that accumulate Phe at a high concentration. Combined application of in vitro mutagenesis for Mtrl gene and synthesis of gene products by wheat-embryo cell-free system could be an efficient approach as proven in the development of the mutated OASA2 enzyme that exhibited both increased enzyme activity and relaxed feedback sensitivity. Since amino acids serve as building blocks for various bioactive compounds, the establishment of a repertoire of genetically engineered plants for accumulation of amino acids is the basis for future development of transgenic plants with new biochemical characters.

\section{Acknowledgements}

We are grateful to our coworkers on our projects. We thank Dr. Joseph G. Dubouzet (Scion Research) for critical reading of the manuscript. This work was supported by CREST of the Japan Science and Technology Agency (JST) and partially by the Multidisciplinary Research of National Agriculture and Biooriented Research Organization (NARO).

\section{References}

Bak S, Tax FE, Feldmann KA, Galbraith DW, Feyereisen R (2001) CYP83B1, a cytochrome P450 at the metabolic branch paint in auxin and indole glucosinolate biosynthesis in Arabidopsis. Plant Cell 13: 101-111

Bohlmann J, DeLuca V, Eilert U, Martin W (1995) Purification and cDNA cloning of anthranilate synthase from Ruta graveolens: modes of expression and properties of native and recombinant enzymes. Plant $J$ 7: 491-501

Bohlmann J, Lins T, Martin W, Eilert U (1996) Anthranilate synthase from Ruta graveolens. Plant Physiol 111: 507-514

Coruzzi G, Last R (2000) Amino acids. In: Buchanan BB, Gruissem W, Jones RL (eds) Biochemistry \& Molecular Biology of Plants. American Society of Plant Physiologists, Maryland, 
pp 358-410

Dixon RA, Paiva NL (1995) Stress-induced phenylpropanoid metabolism. Plant Cell 7: 1085-1097

Dubouzet JG, Ishihara A, Matsuda F, Miyagawa H, Iwata H, Wakasa K (2007) Integrated metabolomic and transcriptomic analyses of high-tryptophan rice expressing a mutant anthranilate synthase alpha subunit. J Exp Bot 58: 3309-3321

Frey M, Chomet P, Glawischnig E, Stettner C, Grün S, et al. (1997) Analysis of a chemical plant defense mechanism in grasses. Science 277: 696-699

Fukuchi A, Fueki T, Kikuchi F, Wakasa K (1990) Differences in several characteristics of rice 5-methyltryptophan resistant mutants selected in tissue culture. Bull Agr \& For Res $U$ Tsukuba 2: 1-11 (in Japanese)

Glawischnig E, Hansen BG, Olsen CE, Halkier BA (2004) Camalexin is synthesized from indole-3-acetaldoxime, a key branching point between primary and secondary metabolism in Arabidopsis. Proc Natl Acad Sci USA 101: 8245-8250

Goers SK, Jensen RA (1984) The differential allosteric regulation of two chorismate-mutses isoenzymes of Nicotiana silvestris. Planta 162: 117-124

Hall GC, Flick MB, Gherna RL, Jensen RA (1982) Biochemical diversity for biosynthesis of aromatic amino acids among the cyanobacteria. J Bacteriol 149: 65-78

Herrmann KM (1995) The shikimate pathway as an entry to aromatic secondary metabolism. Plant Physiol 107: 7-12

Hull AK, Vij R, Celenza JL (2000) Arabidopsis cytochrome P450s that catalyze the first step of tryptophan-dependent indole-3acetic acid biosynthesis. Proc Natl Acad Sci USA 97: 23792384

Im SW, Pittard J (1971) Phenylalanine biosynthesis in Escherichia coli K-12: mutants derepressed for chorismate mutase Pprephenate dehydratase. J Bacteriol 106: 784-790

Ishihara A, Asada Y, Takahashi Y, Yabe N, Komeda Y, Nishioka T, Miyagawa H, Wakasa K (2006) Metabolic changes in Arabidopsis thaliana expressing the feedback-resistant anthranilate synthase $\alpha$ subunit gene OASA1D. Phytochemistry 67: $2349-2362$

Ishihara A, Hashimoto Y, Tanaka C, Dubouzet JG, Nakano T, Matsuda F, Nishioka T, Miyagawa H, Wakasa K (2008) The tryptophan pathway is involved in the defense responses of rice against pathogenic infection via serotonin production. Plant $J$ 54: 481-495

Ishihara A, Matsuda F, Miyagawa H, Wakasa K (2007) Metabolomics for metabolically manipulated plants: effects of tryptophan overproduction. Metabolomics 3: 319-334

Ishihara A, Ohtsu Y, Iwamura H (1999) Biosynthesis of oat avenanthramide phytoalexins. Phytochemistry 50: 237-242

Ishimoto M, Shaikh MR, Mutasim MK. Hany AE, Nakamoto Y, et al. (2009) Evaluation of amino acid profiles and nutritive quality of transgenic soybean seeds accumulating high-level tryptophan. Mol Breed DOI 10.1007/s11032-009-9334-3

Jung E, Zamir LO, Jensen RA (1986) Chloroplasts of higher plants synthesize L-phenylalanine via L-arogenate. Proc Natl Acad Sci USA 83: 7231-7235

Kang S, Kang K, Lee K, Back K (2007) Characterization of rice tryptophan decarboxylases and their distinct involvement in serotonin biosynthesis in transgenic rice. Planta 227: 263-272

Kanno T, Kasai K, Ikejiri-Kanno Y, Wakasa K, Tozawa Y (2004) In vitro reconstitution of rice anthranilate synthase: distinct functional properties of the $\alpha$ subunits OASA1 and OASA2. Plant Mol Biol 54: 11-23
Kanno T, Komatsu A, Kasai K, Dubouzet JG, Sakurai M, IkejiriKanno Y, Wakasa K, Tozawa Y (2005) Structure-based in vitro engineering of the anthranilate synthase, a metabolic key enzyme in the plant Trp pathway. Plant Physiol 138: 22602268

Komatsu A, Ohtake M, Hasegawa H, Terakawa T, Wakasa K (2006) Transgenic rice for animal feed with high tryptophan content generated by a selectable marker- and vector backbonefree technology. Plant Biotechnol 23: 39-46

Kuroki GW, Conn EE (1989) Differential activities of chorismate mutase isozymes in tubers and leaves of Solanum tuberosum L. Plant Physiol 89: 472-476

Li J, Last RL (1996) The Arabidopsis thaliana trp5 mutant has a feedback-resistant anthranilate synthase and elevated soluble tryptophan. Plant Physiol 110: 51-59

Ly D, Kang K, Choi J-Y, Ishihara A, Back K, Lee S-G (2008) HPLC analysis of serotonin, tryptamine, tyramine, and the hydroxycinnamic acid amides of serotonin and tyramine in food vegetables. J Med Food 11: 385-389

Matsuda F, Ishihara A, Takanashi K, Morino K, Miyazawa H, Wakasa K, Miyagawa H (2009) Metabolic profiling analysis of genetically modified rice seedlings that overproduce tryptophan reveals the occurrence of its inter-tissue translocation. Plant Biotechnol (in press)

Matsuda F, Wakasa K, Miyagawa H (2007) Metabolic flux analysis in plants using dynamic labeling technique: Application to tryptophan biosynthesis in cultured rice cells. Phytochemistry 68: 2290-2301

Mayama S, Tani T, Matsuura Y, Ueno T, Fukami H (1981) The production of phytoalexins by oat in response to crown rust, Puccinia coronata f. sp. avenae. Physiol Plant Pathol 19: 217226

Mikkelsen MD, Hansen CH, Wittstock U, Halkier BA (2000) Cytochrome P450 CYP79B2 from Arabidopsis catalyzes the conversion of tryptophan to indole-3-acetaldoxime, a precursor of indole glucosinolate and indole-3-acetic acid. J Biol Chem 275: 33712-33717

Mobley E, Kunkel BN, Keith B (1999) Identification, characterization and comparative analysis of a novel chorismate mutase gene in Arabidopsis thaliana. Gene 240: 115-123

Morino K, Matsuda F, Miyazawa H, Sukegawa A, Miyagawa M, Wakasa K (2005) Metabolic profiling of tryptophanoverproducing rice calli that express a feedback-insensitive $\alpha$ subunit of anthranilate synthase. Plant Cell Physiol 46: 514521

Nester EW, Jensen RA (1966) Control of aromatic acid biosynthesis in Bacillus subtilis: sequential feedback inhibition. J Bacteriol 91: 1594-1598

Nicholson RL, Hammerschmidt R (1992) Phenolic compounds and their role in disease resistance. Ann Rev Phytopathol 30: 369389

Niemeyer HM (1988) Hydroxamic acids (4-hydroxy-1,4benzoxazin-3-ones): defense chemicals in the Gramineae. Phytochemistry 27: 3349-3358

Niyogi KK, Fink GR (1992) Two anthranilate synthase genes in Arabidopsis: defenserelated regulation of the tryptophan pathway. Plant Cell 4: 721-733

Nomura T, Ishihara A, Imaishi H, Ohkawa H, Endo TR, Iwamura H (2003) Rearrangement of the genes for the biosynthesis of benzoxazinones in the evolution of Triticeae species. Planta 217: 776-782

O’Donovan D, Leete E (1963) Biosynthesis of gramine: feeding 
experiments with tryptophan- $\beta$-[H3,C14]. J Am Chem Soc 85 : $461-463$

Poulsen C, Bongaerts RJM, Verpoorte R (1993) Purification and characterization of anthranilate synthase from Catharanthus roseus. Eur J Biochem 212: 431-440

Ramaih S, Guedira M, Paulsen GM (2003) Relationship of indoleacetic acid and tryptophan to dormancy and preharvest sprouting of wheat. Funct Plant Biol 30: 939-945

Rippert P, Matringe M (2002) Molecular and biochemical characterization of an Arabidopsis thaliana arogenate dehydrogenase with two highly similar and active protein domains. Plant Mol Biol 48: 361-368

Sepulveda BA, Corcuera LJ (1990) Effect of gramine on the susceptibility of barley leaves to Pseudomonas syringae. Phytochemistry 29: 465-467

Siehl DL, Conn EE (1988) Kinetic and regulatory properties of arogenate dehydratase in seedlings of Sorghum bicolor (L.) Moench Arch Biochem Biophys 260: 822-829

Song HS, Brotherton JE, Gonzales RA, Widholm JM (1998) Tissue culture-specific expression of a naturally occurring tobacco feedback-insensitive anthranilate synthase. Plant Physiol 117: 533-543

Tozawa Y, Hasegawa H, Terakawa T, Wakasa K (2001) Characterization of rice anthranilate synthase $\alpha$-subunit genes $O A S A 1$ and $O A S A 2$. Tryptophan accumulation in transgenic rice expressing a feedback-insensitive mutant of OASA1. Plant Physiol 126: 1493-1506

Tsuji J, Jackson EP, Gage DA, Hammerschmidt R, Somerville SC (1992) Phytoalexin accumulation in Arabidopsis thaliana during the hypersensitive reaction to Pseudomonas syringae pv syringae. Plant Physiol 98: 1304-1309

Ueno M, Shibata H, Kihara J, Honda Y, Arase S (2003) Increased tryptophan decarboxylase and monoamine oxidase activities induce Sekiguchi lesion formation in rice infected with Magnaporthe grisea. Plant J 36: 215-228

Wakasa K, Hasegawa H, Nemoto H, Matsuda F, Miyazawa H, et al. (2006) High-level tryptophan accumulation in seeds of transgenic rice and its limited effects on agronomic traits and seed metabolite profile. J Exp Bot 57: 3069-3078

Wakasa K, Widholm JM (1987) A 5-methyltryptophan resistant rice mutant, MTR1, selected in tissue culture. Theor Appl Genet 74: 49-54

Wakasa K, Widholm JM (1991) Rice mutants resistant to amino acids and amino acid analogs. In: Bajaj YPS (ed) Biotechnology in Agriculture and Forestry 14 Rice. Springer-Verlag, Berlin Heidelberg, pp 304-315

Warpeha KM, Lateef SS, Lapik Y, Anderson MB, Lee BS, Kaufman LS (2006) G-protein-coupled receptor1, G-protein $\mathrm{G} \alpha$-subunit1, and prephenate dehydratase 1 are required for blue light-induced production of phenylalanine in etiolated Arabidopsis. Plant Physiol 140: 844-855

Widholm JM (1972) Cultured Nicotiana tabacum cells with an altered anthranilate syntase which is less sensitive to feedback inhibition. Biochim Biophys Acta 261: 52-58

Yamada T, Matsuda F, Kasai K, Fukuoka S, Kitamura K, Tozawa Y, Miyagawa H, Wakasa K (2008) Mutation of a rice gene encoding a phenylalanine biosynthetic enzyme results in accumulation of phenylalanine and tryptophan. Plant Cell 20: 1316-1329

Yamada T, Tozawa Y, Hasegawa H, Terakawa T, Ohkawa Y, Wakasa K (2004) Use of a feedback-insensitive $\alpha$ subunit of anthranilate synthase as a selectable marker for transformation of rice and potato. Mol Breed 14: 363-373 